\title{
Novel approach for hepatic flexure mobilization with infracolic, suprapancreatic, medial-to-lateral approach
}

\author{
T. Petropoulou' · S. Amin ${ }^{1}$
}

Received: 28 April 2019 / Accepted: 17 July 2019 / Published online: 20 August 2019

(c) Springer Nature Switzerland AG 2019

\section{Introduction}

Hepatic flexure mobilization can be challenging and most of the time requires patient repositioning.

We present our standardized, novel approach for complete mobilization of the hepatic flexure, which can be used during both laparoscopic and robotic right hemicolectomy, using an infracolic, medial-to-lateral, suprapancreatic approach. This method does not require repositioning of the patient.

The attached video demonstrates a laparoscopic procedure, but the exact same steps are used for our robotic procedures too.

This method has not been previously described.

\section{Technique}

The patient is flat or in slight Trendelenburg position and tilt on the left.

We always position the greater omentum below the liver, so it acts as a retractor.

We then position the small bowel loops to expose the ileocolic pedicle and the superior mesenteric vein (SMV).

We start the dissection parallel to SMV, near the origin of ileocolic vein, and develop the plane.

We first take down ileocolic vein and artery (high ligation between clips) and then continue the medial dissection with a Kocher Maneuver till we expose the 2nd-3rd part of the duodenum and the head of the pancreas.

Continuing in this infracolic suprapancreatic plane, we separate the mesocolon from retroperitoneal structures.

Electronic supplementary material The online version of this article (https://doi.org/10.1007/s10151-019-02047-1) contains supplementary material, which is available to authorized users.

T. Petropoulou

thalia_pet@hotmail.com

1 Sheffield Teaching Hospitals, Sheffield, UK
We continue the dissection until we break through to the gallbladder fossa and the lateral abdominal wall.

Then, we proceed until the hepatic flexure is fully mobile from below.

The last few lateral attachments and the gastrocolic omentum are taken down.

The laparoscopic part of the procedure is done, the specimen is taken out and we then perform the anastomosis, which, for laparoscopic procedures is an extracorporeal, stapled, side-to-side isoperistaltic ileocolic anastomosis.

The advantages of this method are that it does not require a steep head down (which could be contraindicated in specific groups of patients), does not require patient repositioning (or dual docking for robotic procedures), and could help with difficult hepatic flexure tumors, where the planes near the tumor are fused.

\section{Compliance with ethical standards}

Conflict of interest The authors declare that they have no conflict of interest.

Ethical approval Ethical approval has been obtained from our institution and clinical and effectiveness department and the study was conducted according to 1964 Helsinki good clinical practice guidelines.

Informed consent Informed consent was obtained from all participants.

Publisher's Note Springer Nature remains neutral with regard to jurisdictional claims in published maps and institutional affiliations. 